\title{
Effect of particle shape on the efficiency of granular dampers
}

\author{
Hamzeh Pourtavakoli ${ }^{1, \star}$, Eric J. R. Parteli ${ }^{2, \star \star}$, and Thorsten Pöschel ${ }^{1, \star \star \star}$ \\ ${ }^{1}$ University of Erlangen-Nuremberg, Erlangen, Germany \\ ${ }^{2}$ University of Cologne, Cologne, Germany
}

\begin{abstract}
Granular dampers, containers partially filled with granular material, are often applied for attenuating mechanical vibrations in a broad range of systems. However, the role of the particle shape on the performance of the damper has remained largely uncertain and is investigated here by means of particle-based simulations. It is found that, for large excitation amplitudes (collect-and-collide regime), particle shape nearly does not affect the damper's performance. For low excitation amplitudes (gas-like regime), a dependence on the average dissipated energy per cycle on the particle shape is found. In this regime, the spherical particle geometry leads to the highest damper's efficiency.
\end{abstract}

\section{Introduction}

Many authors have studied the influence of the parameters of the driving $[1,2]$, material properties and particle size [3-6] on the performance of granular dampers. However, the role of particle shape has remained largely out of the focus of previous investigations. This role is examined in the present work by means of numerical simulations using the Discrete Element Method (DEM), which consists of simultaneously solving Newton's equations of translational and rotational motion for all particles as described in detail in Ref. [7]. We used DEM to perform a systematic study by investigating a diversity of particle shapes covering a broad range of shape parameter values (specified below).

Since granular dampers perform particularly well in applications where the acceleration due to gravity can be neglected (as gravity tends to demobilize the granulate [1]), we focus here mainly on the study of granular dampers in microgravity conditions.

\section{Numerical experiments}

Although most DEM models are designed for particulate systems composed of spherical particles, geometrically complex particles can be modeled using the multisphere method, where spherical particles are arranged to form composite particles [8-11]. In Table 1 we present different particle shapes chosen to cover a broad range of values of the following shape parameters [12, 13]:

- aspect ratio, that is, the ratio of the Feret's minimum diameter to the Feret's maximum diameter

$$
\eta \equiv D_{\min }^{\mathrm{F}} / D_{\max }^{\mathrm{F}},
$$

^e-mail: hamzeh.pourtavakoli@fau.de

$\star \star$ e-mail: eric.parteli@uni-koeln.de

$\star \star \star$ e-mail: thorsten.poeschel@fau.de
Table 1. Multisphere particles used in the numerical experiments (first row) along with associated symbols (second row) used in figs. 2-4. The corresponding shape parameters are listed in the subsequent rows. For particles \#2 - \#11 these parameters are calculated with respect to the plane $i-j$ (see coordinate system on the bottom right hand corner). Bottom: particle \#8 with the spherocylinders encompassing the particle's constituent beads (first sketch from left to right) as well as the definition of $P, A, P_{\mathrm{c}}$ and $A_{\mathrm{c}}$ for this particle.

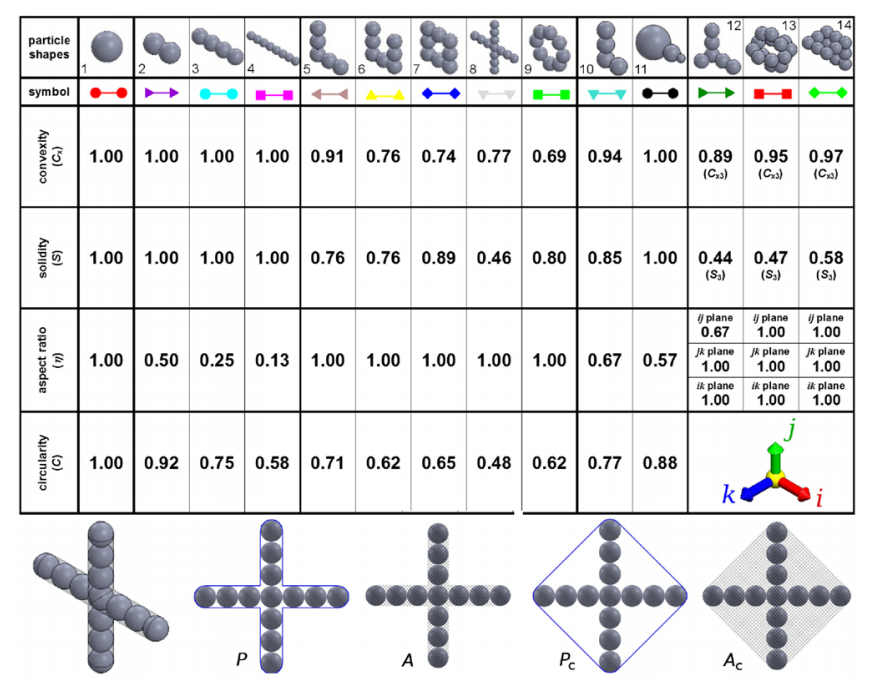

- circularity,

$$
C \equiv \sqrt{4 \pi A / P^{2}},
$$

where $A$ and $P$ denote the area and perimeter of the particle, respectively.

- convexity

$$
C_{\mathrm{x}} \equiv P_{\mathrm{c}} / P
$$


where $P_{\mathrm{c}}$ is the hull perimeter.

- solidity,

$$
S \equiv A / A_{\mathrm{c}},
$$

where $A_{\mathrm{c}}$ is the convex hull area. That is, $A_{\mathrm{c}}$ denotes the area inside the convex hull around the two-dimensional particle shape.

The sketches at the bottom of Table 1 show how to define $P, P_{\mathrm{c}}, A$ and $A_{\mathrm{c}}$ taking as example particle \#8.

Moreover, for particle shapes \#12 - \#14 the centerof-mass positions of the constitutent beads do not lie on the same plane, that is, these complex particles are threedimensional objects. Therefore, for these particles, the convexity and solidity are defined as follows:

- convexity,

$$
C_{\mathrm{x} 3}=A_{\mathrm{c} 3} / A_{3},
$$

- solidity,

$$
S_{3}=V_{3} / V_{\mathrm{c} 3},
$$

where $A_{3}\left(V_{3}\right)$ is the sum of the total surface area (volume) of all spherocylinders enclosing the constituent spheres of the composite particle, while $A_{\mathrm{c} 3}\left(V_{\mathrm{c} 3}\right)$ is the area (volume) of the three-dimensional convex hull or the smallest three-dimensional envelope enclosing the particle shape. Moreover, while the circularity is a measure inherent to two-dimensional objects [13], the aspect ratio of particle shapes \#12 - \#14 can be computed for each one of the planes defined in Table 1 and the results are displayed in this table.

In the DEM model, the multispheres particles are treated as rigid bodies, the translational and rotational motion of which results from the forces acting on the constituent beads. These forces are computed using noncohesive inter-particle force model based on Cundall and Strack [14] and described in detail elsewhere [15]. In the model, the beads are treated as viscoelastic and friction is neglected [15]. The model equations have been implemented in the DEM library of Ref. [16], and the values of the model parameters are listed in table 2 (see Ref. [15] for more details about the model).

Table 2. Numerical values of the parameters used in the simulations. For particle-wall collisions the Poisson's ratio used

was $v=0.37$. The dimensions of the damper $(L, W$ and $H$, defined with respect to the axes $x, y$ and $z$, respectively) are as in Ref. [17].

\begin{tabular}{lcl}
\hline parameter & symbol & value \\
\hline particle material density & $\rho_{\mathrm{p}}$ & $7800 \mathrm{~kg} / \mathrm{m}^{3}$ \\
particle diameter & $d$ & $4 \mathrm{~mm}$ \\
Young's modulus & $Y$ & $10^{8} \mathrm{~Pa}$ \\
Poisson's ratio & $v$ & 0.3 \\
box length & $L$ & $100 \mathrm{~mm}$ \\
box width & $W$ & $50 \mathrm{~mm}$ \\
box height & $H$ & $50 \mathrm{~mm}$ \\
timestep & $\Delta t$ & $10^{-6} \mathrm{~s}$ \\
\hline
\end{tabular}

\section{Results and discussion}

Figure 1 shows snapshots of the simulations of the damper oscillation. As we can see from this figure, for an amplitude of $10 \mathrm{~mm}$, the granular system is in a gas-like regime, in which only a few particles interact with the oscillating walls during one oscillation period [17]. By contrast, for the amplitude of $80 \mathrm{~mm}$, the system is in the collect-andcollide regime [17]. These regimes are separated by an optimal operation mode - the critical amplitude of the damper's oscillation - for which the energy dissipation is maximal.

The damping efficiency associated with a given particle shape can be characterized by computing the relative energy dissipated per cycle [17], $E_{\mathrm{diss}} / E_{\max }$, and is shown in Fig. 2 as a function of the oscillation amplitude for all particle shapes investigated. As we can see in this figure, for all particle shapes, the value of damping efficiency ( $\left.E_{\text {diss }} / E_{\max }\right)$ increases linearly with the amplitude $A_{\text {damp }}$ in the gas-like regime, for $0<A_{\text {damp }} \lesssim 20 \mathrm{~mm}$. Moreover, for $A_{\text {damp }}=A_{\text {crit }} \approx 30 \mathrm{~mm}$ a maximal value is reached, whereas the system enters the collect-and-collide regime as the amplitude becomes larger than $30 \mathrm{~mm}$. As can be seen from Fig. 2, the critical amplitude $A_{\text {crit }}$ is nearly independent of the particle shape. We also see in Fig. 2 that the results for the different particle shapes collapse well for the collect-and-collide regime. In this regime, the behavior of the system can be described by by means of a one-particle model [18, 19].

For amplitudes in the gas-like regime (in particular when $A_{\text {damp }} \ll A_{\text {crit }}$ ), we observe an interesting behavior in Fig. 2, that is the curves do not collapse as well as they do in collect-and-collide regime $\left(A_{\text {damp }}>A_{\text {crit }}\right)$. In the gas-like regime, we expect the dissipative behavior of the system to be related with the granular temperature $[7,20]$, or equivalently the spread of the granular system, since in this regime the bulk of particles is located around the center of the damper (thus far from the driving walls). This means that the dissipated energy is due to collisions of only a few particles with driving walls, which are far away from the bulk.

The simplest quantity which we can compute to investigate this behavior is the standard deviation of the positions of the particles along the oscillation axis relative to the particles' center-of-mass position. We thus investigate the time-averaged standard deviation of the particles' positions $x_{i}(t)$ relative to the center-of-mass position $x_{\mathrm{cm}}(t)$ of the granular material, $\sigma_{x}=\left\langle\sum_{i=1}^{N}\left(x_{i}(t)-x_{\mathrm{cm}}(t)\right)^{2} / N\right\rangle_{t}^{1 / 2}$ (with $N=473$ particles), for an amplitude $A_{\text {damp }}=$ $10 \mathrm{~mm}$, which is well within the range of amplitudes characterizing the gas-like regime. Figure 3 shows the damping efficiency as a function of $\sigma_{x}$ for all particle shapes investigated. As we can see, by considering all complex particles shapes investigated, a linear increase of the damping efficiency with $\sigma_{x}$ is observed, whereas this behavior is found for all amplitudes below $15 \mathrm{~mm}$, that is when the system is well within the gas-like regime. The best fit to the simulation data associated with the complex particle shapes using the equation 


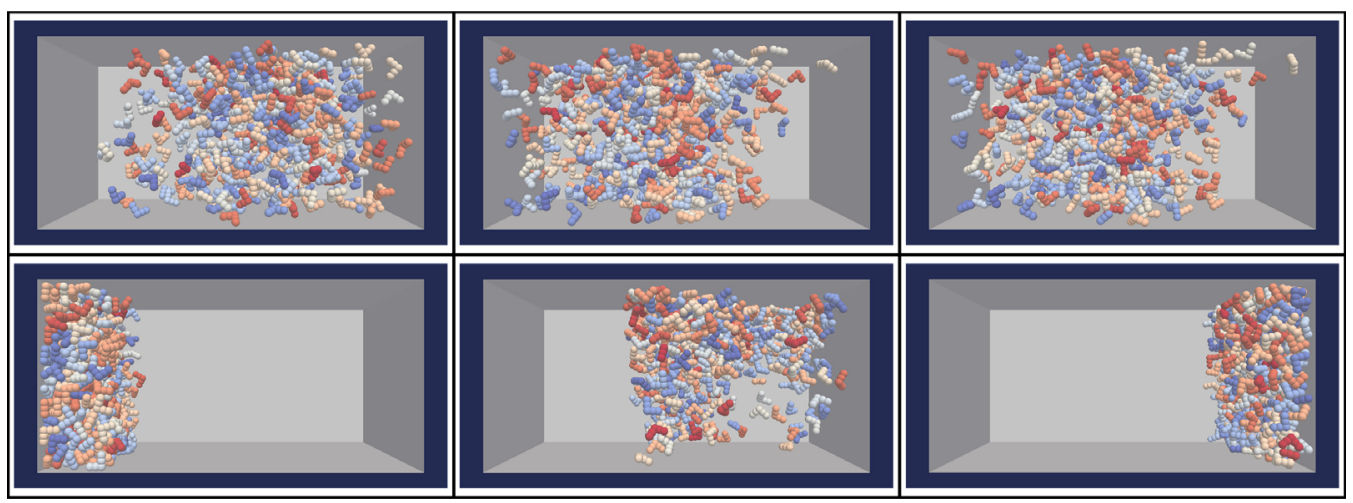

Figure 1. Snapshots of the damper simulations using particle shape \#5 in Fig. 1 at oscillation amplitudes $A_{\text {damp }}=10 \mathrm{~mm}$ (top; gaslike regime) and $A_{\text {damp }}=80 \mathrm{~mm}$ (bottom; collect-and-collide regime). Shown are snapshots corresponding to the oscillation phases $\pi / 2, \pi$ and $3 \pi / 2$ (from left to right). In the simulations, the damper's horizontal position evolves in time according to the equation $x(t)=A_{\mathrm{damp}} \sin (2 \pi v t)($ where $v=4 \mathrm{~Hz}$ is the oscillation frequency [17] $)$.

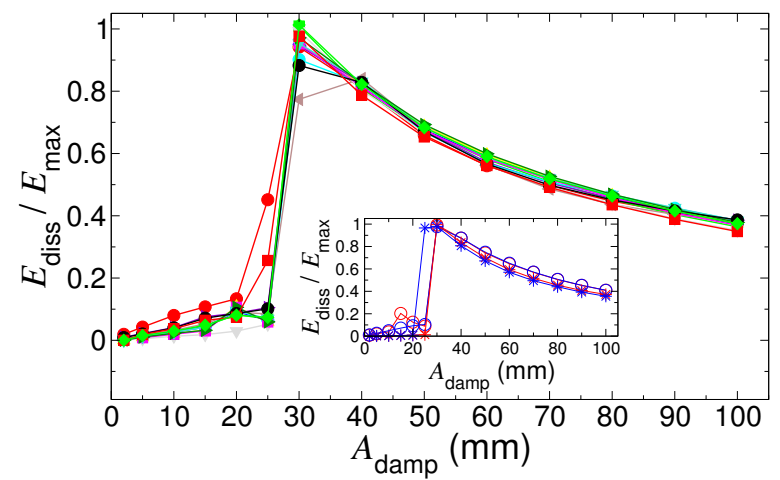

Figure 2. The main plot shows the damping efficiency, $E_{\mathrm{diss}} / E_{\mathrm{max}}$, as a function of the oscillation amplitude. The symbols correspond to different particle shapes as defined in Table 1. The inset shows the corresponding results for particles \#1 (circles) and \#4 (stars) of Table 1 obtained from simulations with frictional interactions, with $\mu=0.2$ (red) and $\mu=0.5$ (blue).

$$
\frac{E_{\mathrm{diss}}}{E_{\max }}=a+b \sigma_{x}
$$

gives $a \approx-0.0688$ and $b \approx 0.00565 \mathrm{~mm}^{-1}$, with correlation coefficient 0.95 . This best fit is denoted by the continuous line in Fig. 3.

By considering one type of particle shape (the rods), we can see a dependence of the damping efficiency on some of the shape parameters. We see in Fig. 4a that the damping efficiency increases linearly with the aspect ratio $\eta$ of the rods, whereas the best fit to the data using the equation $E_{\text {diss }} / E_{\max }=a_{r}+b_{r} \eta$ gives $a_{r} \approx 0.0097$ and $b_{r} \approx 0.069$ with correlation coefficient 0.997 . Moreover, in Fig. 4b we see that the damping efficiency increases non-linearly with circularity $(C)$ of the rods. The best fit using the equation $E_{\text {diss }} / E_{\max }=a_{c}+b_{c} C^{\alpha}$ gives $a_{c} \approx 0.023, b_{c} \approx 0.057$ and $\alpha \approx 13.2$ with correlation coefficient 0.994 .

While the present manuscript focuses on the damping efficiency based on the conditions of the experiments of

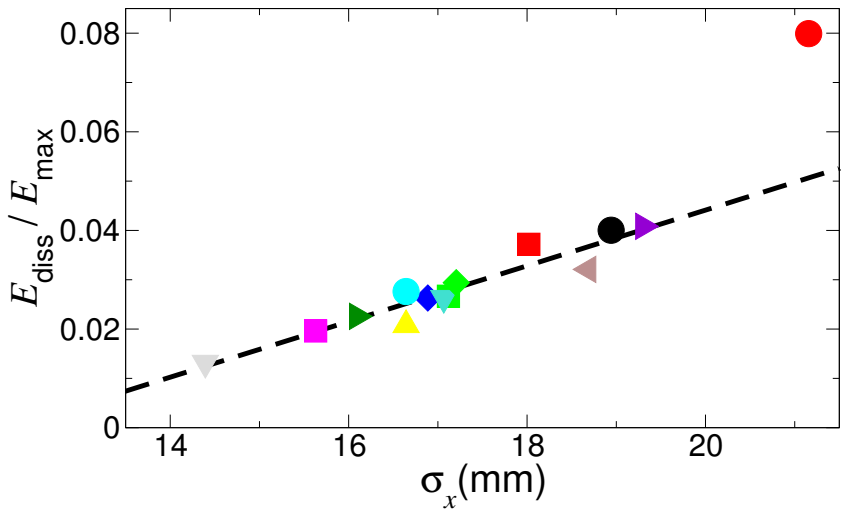

Figure 3. Dependence of the damping efficiency on the timeaveraged standard deviation of the particles' positions along the oscillation axis $(x)$ relative to the center-of-mass position of the particulate system. An amplitude of oscillation $A_{\text {damp }}=10 \mathrm{~mm}$ (gas-like regime) has been considered. Particle shapes are denoted to different symbols as in Table 1. The dashed line represents the fit to the simulation data (with exception of the spherical particle) using Eq. (7).

Refs. [17, 18, 21], the physics of the granular system in the gas-like regime should be investigated more systematically considering different particle numbers and system sizes. Moreover, it would be interesting to investigate the dissipative behavior of the different particle shapes in more detail, especially close to the critical amplitude where dissipation is maximal (see Fig. 2).

\section{Conclusions}

By means of DEM simulations under consideration of complex particle shapes, we have shown that particle shape affects the performance of granular dampers in the regime where the amplitude of oscillation is low (gas-like regime). In this regime, we could find an increase of damper's efficiency with circularity and aspect ratio when the particle shape is the rod. Moreover, independently of 


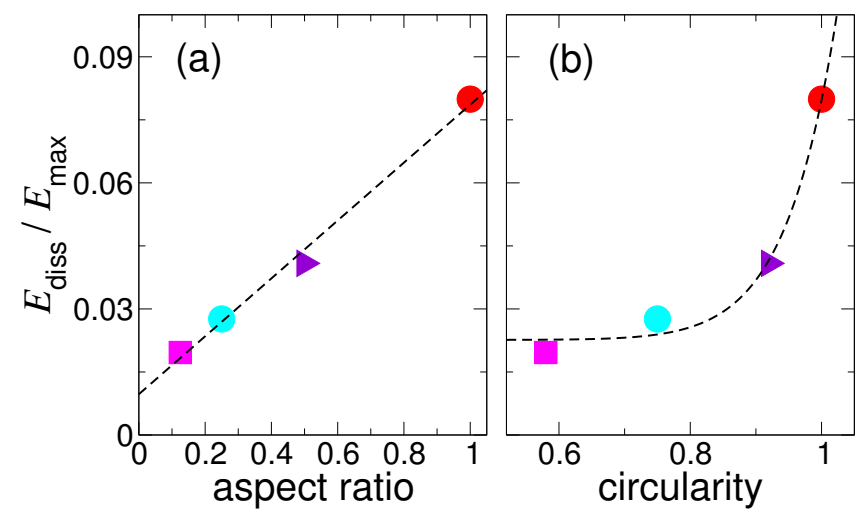

Figure 4. Damping efficiency $E_{\text {diss }} / E_{\max }$ as a function of aspect ratio $\eta$ (a) and circularity $C$ (b) for the rods. An amplitude of oscillation $A_{\text {damp }}=10 \mathrm{~mm}$ (gas-like regime) has been considered. The symbols denote different particle shapes according to Table 1. The dashed lines correspond to the fits $E_{\text {diss }} / E_{\max }=$ $0.0097+0.069 \eta$ (a) and $E_{\text {diss }} / E_{\max }=0.023+0.057 C^{13.2}$ (b).

the particle shape, we found that the average energy dissipated by cycle of the damper in the gaseous regime increases with the standard deviation of the particle's position. This can be understood by noting that this standard deviation is a measure of the granular temperature, which plays an important role for the system's dynamics. Interestingly, the spherical particle shape leads to the highest efficiency in the gas-like regime.

Furthermore, we found that the critical amplitude above which the system abandons the gas-like regime and enters the collect-and-collide regime is independent of the particle shape. Moreover, in the collect-and-collide regime, the dependence of the damper's efficieny on the amplitude of oscillation is nearly independent of the particle shape.

Based on our results, the following question is raised: Does particle shape matter for the damper's efficiency? As a matter of fact, most dampers operate with nearly spherical particles, and our simulations suggest no improvement in the damper's efficiency is obtained if complex particles are used. This work should be now continued by including effect of friction. However, we have obtained results for spheres and rods using Coulomb friction coefficients $\mu=0.2$ and $\mu=0.5$, from which we found little dependence of $E_{\text {diss }} / E_{\max }$ on $\mu$ (see Fig. 2). Moreover, the effect of cohesion following the model of Ref. [22] as well as particle material properties (stiffness and normal dissipation behavior) on the damping efficiency should be investigated in future work.

\section{Acknowledgements}

We thank Mohammad Farahani for discussions. We thank the German Research Foundation (DFG) for funding through the Cluster of Excellence "Engineering of Advanced Materials", ZISC, FPS, the Collaborative Research Center SFB814, and grant PO472/20-2. We grate- fully acknowledge the computing time granted by the John von Neumann Institute for Computing (NIC) and provided on the supercomputer JUROPA at Jülich Supercomputing Centre (JSC).

\section{References}

[1] C. Salueña, T. Pöschel and S. E. Esipov, Phys. Rev. E 59, 4422-4425 (1995)

[2] C. Salueña, S. E. Esipov, T. Pöschel and S. Simonian, Proc. SPIE 3327, 19-26 (1998)

[3] W. Chen, M. Hou, K. Lu, Z. Jiang and L. Lam, Phys. Rev. E 64, 061305 (2001)

[4] K. S. Marhadi and V. K. Kinra, J. Sound Vib. 283, 433-448 (2005)

[5] X. M. Bai, L. M. Keer, Q. L. Wang and R. Q.Snurr, Granular Matter 11, 417-429 (2009)

[6] M. Sánchez, G. Rosenthal and L. A. Pugnaloni, J. Sound Vib. 331, 4389-4394 (2012)

[7] T. Pöschel and T. Schwager, Computational Granular Dynamics (Springer, Heidelberg, 2005)

[8] T. Pöschel and V. Buchholtz, Phys. Rev. Lett. 71, 3964-3966 (1993)

[9] J. A. C. Gallas and S. Sokołowski, Int. J. Mod. Phys. 7, 2037-2046 (1993)

[10] E. J. R. Parteli, AIP Conf. Proc. 1542, 185-188 (2013)

[11] E. J. R. Parteli and T. Pöschel, Powder Technology 288, 96-102 (2016)

[12] ISO (2008), ISO 9276-6:2008, Representation of results of particle size analysis - Part 6: Descriptive and quantitative representation of particle shape and morphology (ISO, Geneva, 2008)

[13] E. Olson, Journal of GXP Compliance 15(3), 85-96 (2011)

[14] P. A. Cundall and O. D. L. Strack, Geotechnique 29, 47-65 (1979)

[15] H. Pourtavakoli, E. J. R. Parteli and T. Pöschel, New J. Phys. 18, 073048 (2016)

[16] C. Kloss, C. Goniva, A. Hager, S. Amberger and S. Pirker, Prog. Comput. Fluid Dy. 12, 140-152 (20112)

[17] A. Sack, M. Heckel, J. Kollmer, F. Zimber and T. Pöschel, Phys. Rev. Lett. 111, 018001 (2013)

[18] M. N. Bannerman, J. Kollmer, A. Sack, M. Heckel, P. Müller and T. Pöschel, Phys. Rev. E 84, 011301 (2011)

[19] A. Sack, M. Heckel, J. Kollmer and T. Pöschel, Granular Matter 17, 73-82 (2015)

[20] K. Harth, U. Kornek, T. Trittel, U. Strachauer, S. Hörne, K. Will and R. Stannarius, Phys. Rev. Lett. 110, 144102 (2013)

[21] J. Kollmer, M. Tupy, M. Heckel, A. Sack and T. Pöschel, Phys. Rev. Applied 3, 024007 (2015)

[22] E. J. R. Parteli, J. Schmidt, C. Blümel, K.-E. Wirth, W. Peukert and T. Pöschel, Scientific Reports 4, 6227 (2014) 